\title{
A Clinical Study of Pathadi Yoga and Piccha Basti in the Management of Raktarsha Roga
}

\author{
Dr. Dharmendra Kumar Sharma \\ Reader, Deptt. Of Kayachikitsa, SBLD Ayurved \\ vishwabharti, sardar shahar, Churu, Rajasthan.
}

\author{
Dr. Naresh Kumar Kumawat \\ Lecturer, Deptt. Of Kayachikitsa, SBLD Ayurved \\ vishwabharti, sardar shahar, Churu, Rajasthan.
}

Prof. Rajyavardan Singh Roy

Ex. Prof. and Head, Deptt. Of Kayachikitsa, MMM Govt. Ayurvdic College, Udaipur, Rajasthan.

\begin{abstract}
The prevalence of piles the worldwide. It may occur at any age but mostly seen at the age between 30 to 65 years, equally susceptible in both the sexes. Arsha (Haemorrhoids) is a very common clinical condition affecting half of world population by the age of 50 and out of these half of the patients are suffering from bleeding piles (Raktarsha). The trial was comparative, open and randomized divided in four groups (group $\boldsymbol{A}$ given pathadi yoga $3 \mathrm{gms}$ twice a day with cold water for two months, group $\boldsymbol{B}$ given piccha basti $400 \mathrm{ml}$ daily in morning before meal for 10 days, group $C$ given piccha anuvasna basti $50 \mathrm{ml}$ daily in morning before meal for 10 days and group $\boldsymbol{D}$ given pathadi yoga 3 gms twice a day with cold water for 40 days and piccha anuvasna basti $50 \mathrm{ml}$ daily in morning before meal for 10 days in 10 patients of each groups. After complete of trial work, to find out the efficacy of these trial drugs that Pathadi yoga group got $53.88 \%$ relief, Piccha basti group got $61.33 \%$ relief, Piccha anuvasan group got $55.70 \%$ and group D (pathadi yoga and piccha anuvasan basti) got $65.26 \%$ relief in all symptoms of raktarsha. So concluded that trial drug in combination were statistically effective in bleeding piles.
\end{abstract}

Keywords: Raktarsha, Pathadi yoga, Piccha basti, Piccha anuvasana basti.

\section{INTRODUCTION}

The prevalence rate of piles is $4.4 \%$ in the world in about 10 million people. It may occur at any age but mostly seen at the age between 30 to 65 years, equally susceptible in both the sexes. Arsha is named as a disease which kills the patient like an enemy ${ }^{1}$. Aacharya charaka says it is originated due to vitiation of mamsa dhatu ${ }^{2}$. Raktarsha is one of the six types of $a r s h a^{3}$. Haemorrhoids arise from congestion of the internal and or external venous plexuses around the anal canal. They are extremely common in adults ${ }^{4}$. Symptomatic haemorrhoids affect more than one million individuals in the Western world per year. It is estimated that approximately one half of all Americans have had this condition by the age of 50 , and that $50 \%$ to $85 \%$ of the world's population will be affected by hemorrhoids at some time in their life. However, only a small number seek medical treatment. Annually, only about 500,000 in the U.S. are medically treated for massive hemorrhage, with 10 to $20 \%$ of them requiring surgeries. Haemorrhoids are common in economically developed communities but nowadays the numbers of cases increasing India too because of massive change in their diet and life style.

\section{AIMS AND OBJECTIVES}

$>$ Critical and Conceptual and clinical analysis of raktarsa in relation with bleeding piles.

$>$ To assess the efficacy of the Pathadi Yoga, piccha basti and piccha anuvasana basti in newlyand chronic case of raktarsa.

To find out the combined effect of Pathadi yoga and Piccha anuvasan basti in raktarsha.

\section{Materials AND Methods}

The study will be conducted on 40 clinically and pathologically diagnosed patients of raktarsa [bleeding piles]. The selection of patients will be made from OPD/IPD of Arogyashala, M.M.M. 
Govt. Ayurveda College, Udaipur (Raj.). Forty participants satisfying the eligibility criteria were divided into for equal groups. Each group contains ten participants.

\subsection{Grouping: Group A}

Pathadi Yoga 3gm BD with cold water for two months, Group B- Piccha basti 400ml daily morning before food for10days, Group C- Piccha anuvasana basti 50ml daily morning after food for 10days, Group D- Pathadi Yoga 3gm BD for 40days and Piccha basti 50ml daily in the morning after meal for 10days. Study Design: Comparative Clinical Trial Sample Size: 40 participants (10 in each group)

\subsection{Exclusion Criteria}

Known cases of diabetes mellitus and pregnancy.

Age less than 16year.

Cases with malignancy and rectal prolapsed.

Patients with third and fourth degree will be excluded.

\subsection{Inclusion Criteria}

Age above 16 years and below 70years.

Chronic cases less than two year.

No sex discrimination.

There are four folds of treatments for arsha, these includes bheshaja, kshara, agni and shastra $k a r m a^{6}$. As most common protocol is medicinal treatment for arsha and as a part of shamana therapy it is planned to manage the raktarsha with two different combinations. One was given through oral route i.e. Pathadi yoga and another was given through anal rout i.e. piccha basti.

Pathadi yoga is well explained by aacharya charaka in chikitsa sthana ${ }^{7}$ this yoga contains six medicines patha, rasanjana and indrayava etc. These drugs are having great role in bleeding disorders. Pathadi yoga is also having an analgesic effect, so this Pathadi yoga was selected for the study by oral route.

Piccha basti is prepared by some especial medicines that include yavasa, kush, nyagrodha, ashvattha, shalmali, mocharasa ${ }^{8}$ etc. These all drugs having stambhana (astringent) properties and widely used in increased intestinal motility. Piccha basti also was selected in both pattern of basti i.e. anuvasana and nirooha for the study.

\subsection{Criteria for Assessment}

Hemorrhoid on 3 O' Clock / 7 O' Clock / 11 O' Clock / Other Position

All symptoms of Raktarsha as per ayurvedic text.
(a) $\mathrm{HB} \%$
(b)T.L.C.
(c)D.L.C.
(d) B.T.
(e) C.T.
(f)E.S.R.
(g) Urine- $\mathrm{R}$ $\mathrm{M}$
(h) Stool - R M.

Overall assessment of all the symptoms of raktarsha:

- Complete relief

$100 \%$

- Marked relief Above $75 \%$

- Moderate Relief $51-75 \%$

- Mild relief $26-50 \%$

- No relief Below $25 \%$ 


\subsection{Collection of Data}

The data was collected from each group before treatment and after treatment. Scoring was given and finally the data was compared and analyzed.

\subsection{Statistical Analysis}

The data was collected before treatment and after treatment. Thus collected data were subjected to statistical analysis by using student's t-test along with consultation of a bio-statistician.

\subsection{Result and Interpretation}

Table1. Symptomatic result in all groups.

\begin{tabular}{|c|c|c|c|c|c|c|c|c|}
\hline \multirow{2}{*}{ Symptoms } & \multicolumn{2}{|c|}{ Group A } & \multicolumn{2}{|c|}{ Group B } & \multicolumn{2}{c|}{ Group C } & \multicolumn{2}{c|}{ Group D } \\
\hline & Mean \pm SD & P value & Mean \pm SD & P value & Mean \pm SD & P value & Mean \pm SD & P value \\
\hline 1 & $1.1 \pm 0.57$ & $<0.001$ & $1.3 \pm 0.67$ & $<0.001$ & $1.8 \pm 0.79$ & $<0.001$ & $1.8 \pm 0.78$ & $<0.001$ \\
\hline 2 & $0.6 \pm 0.52$ & $<0.001$ & $0.7 \pm 0.67$ & $<0.05$ & $2 \pm 0.67$ & $<0.001$ & $1.2 \pm 0.63$ & $<0.001$ \\
\hline 3 & $0.8 \pm 0.63$ & $<0.001$ & $0.4 \pm 0.52$ & $<0.05$ & $0.6 \pm 0.52$ & $<0.001$ & $0.6 \pm 0.51$ & $<0.001$ \\
\hline 4 & $0.8 \pm 0.63$ & $<0.001$ & $0.3 \pm 0.48$ & NS & $0.6 \pm 0.70$ & $<0.05$ & $0.4 \pm 0.69$ & NS \\
\hline 5 & $0.6 \pm 0.52$ & $<0.001$ & $0.2 \pm 0.42$ & NS & $0.4 \pm 0.70$ & NS & $0.3 \pm 0.48$ & NS \\
\hline 6 & $0.6 \pm 0.52$ & $<0.001$ & $0.3 \pm 0.48$ & NS & $0.3 \pm 0.48$ & NS & $0.6 \pm 0.84$ & $<0.05$ \\
\hline 7 & $0.8 \pm 0.42$ & $<0.001$ & $0.8 \pm 0.79$ & $<0.05$ & $0.3 \pm 0.48$ & NS & $0.9 \pm 0.73$ & $<0.001$ \\
\hline 8 & $1.4 \pm 0.70$ & $<0.001$ & $2.1 \pm 0.57$ & $<0.001$ & $1.9 \pm 0.74$ & $<0.001$ & $1.7 \pm 0.67$ & $<0.001$ \\
\hline 9 & $0.1 \pm 0.32$ & NS & $0.2 \pm 0.42$ & NS & $0.1 \pm 0.32$ & NS & $0.3 \pm 0.67$ & NS \\
\hline 10 & $0.1 \pm 0.32$ & NS & $0.2 \pm 0.42$ & NS & $0.9 \pm 0.57$ & $<0.001$ & $0.3 \pm 0.67$ & NS \\
\hline 11 & $0.1 \pm 0.32$ & NS & $0 \pm 0.00$ & NS & $0 \pm 0.00$ & NS & $0 \pm 0$ & NS \\
\hline 12 & $0.5 \pm 0.53$ & $<0.05$ & $0.6 \pm 0.52$ & $<0.001$ & $0.8 \pm 0.63$ & $<0.001$ & $0.4 \pm 0.69$ & NS \\
\hline 13 & $0.2 \pm 0.42$ & NS & $0 \pm 0.00$ & NS & $0.2 \pm 0.42$ & NS & $0.2 \pm 0.42$ & NS \\
\hline 14 & $0.3 \pm 0.48$ & NS & $0.3 \pm 0.67$ & NS & $0.2 \pm 0.42$ & NS & $0.7 \pm 0.48$ & $<0.001$ \\
\hline 15 & $0.6 \pm 0.52$ & $<0.001$ & $0.2 \pm 0.63$ & NS & $0.1 \pm 0.32$ & NS & $0.2 \pm 0.42$ & NS \\
\hline 16 & $0.9 \pm 0.74$ & $<0.001$ & $0.5 \pm 0.71$ & $<0.05$ & $0.4 \pm 0.52$ & $<0.05$ & $1.1 \pm 0.56$ & $<0.001$ \\
\hline 17 & $1.2 \pm 0.42$ & $<0.001$ & $1.2 \pm 0.92$ & $<0.001$ & $1.4 \pm 0.84$ & $<0.001$ & $1 \pm 0.47$ & $<0.001$ \\
\hline 18 & $0.1 \pm 0.32$ & NS & $0.1 \pm 0.32$ & NS & $0.1 \pm 0.32$ & NS & $0.5 \pm 0.52$ & $<0.05$ \\
\hline 19 & $0.3 \pm 0.48$ & NS & $0 \pm 0.00$ & NS & $0.1 \pm 0.32$ & NS & $0.2 \pm 0.42$ & NS \\
\hline
\end{tabular}

$<0.001 \rightarrow$ highly significant

$<0.05 \rightarrow$ Significant

N.S. $\rightarrow$ Non-significant

Table2. showing result in all Symptoms in different groups.

\begin{tabular}{|c|c|c|c|c|c|}
\hline S.No. & Symptoms & Group A & Group B & Group C & Group D \\
\hline 1. & Vibandha(constipation) & $50 \%$ & $60 \%$ & $50 \%$ & $65 \%$ \\
\hline 2. & Gudankura(anorectal pile) & $60 \%$ & $50 \%$ & $55 \%$ & $65 \%$ \\
\hline 3. & Adhmaan(flatulence) & $70 \%$ & $100 \%$ & $25 \%$ & $75 \%$ \\
\hline 4. & Gudakandu(Itching at anal) & $55 \%$ & $50 \%$ & $25 \%$ & $55 \%$ \\
\hline 5. & Gudashotha (anal swelling) & $30 \%$ & $65 \%$ & $30 \%$ & $50 \%$ \\
\hline 6. & Gudadaha (burning sensation) & $45 \%$ & $70 \%$ & $45 \%$ & $75 \%$ \\
\hline 7. & Gudashula (pain) & $30 \%$ & $55 \%$ & $25 \%$ & $70 \%$ \\
\hline 8. & Raktasrava(secretion) & $55 \%$ & $85 \%$ & $55 \%$ & $85 \%$ \\
\hline 9. & Pravahika (mucous secretion) & ----- & $100 \%$ & $100 \%$ & $100 \%$ \\
\hline 10. & Parikartika(cutting type pain) & $100 \%$ & $65 \%$ & $45 \%$ & $100 \%$ \\
\hline 11. & Karshya(weakness) & $75 \%$ & ----- & ----- & ------ \\
\hline 12. & Pandu(anemia) & $65 \%$ & $50 \%$ & $55 \%$ & $45 \%$ \\
\hline 13. & Sangrahni (mucous Secretion) & $50 \%$ & ----- & $100 \%$ & $100 \%$ \\
\hline 14. & Katishula(backache) & $80 \%$ & $35 \%$ & $65 \%$ & $75 \%$ \\
\hline 15. & Udarashula (abdominal pain) & $65 \%$ & $65 \%$ & $30 \%$ & $65 \%$ \\
\hline 16. & Arochaka(anorexia) & $45 \%$ & $55 \%$ & $100 \%$ & $55 \%$ \\
\hline 17. & Agnimandya(indigation) & $60 \%$ & $70 \%$ & $45 \%$ & $50 \%$ \\
\hline 18. & Hatoujasa+( ed Immunity power) & $25 \%$ & $50 \%$ & $100 \%$ & $70 \%$ \\
\hline 19. & Pindikodveshtana(cramps) & $50 \%$ & ----- & $50 \%$ & $65 \%$ \\
\hline
\end{tabular}


Dr. Dharmendra Kumar Sharma et al.

Table:3 -Statistical Analysis by ANOVA Technique

\begin{tabular}{|l|l|l|l|l|l|l|}
\hline & F value & P value & & & F value & P value \\
\hline $\mathbf{1}$ & 3.86 & $<0.05$ & & $\mathbf{1 1}$ & 0.04 & NS \\
\hline $\mathbf{2}$ & 16.10 & $<0.001$ & & $\mathbf{1 2}$ & 3.42 & $<0.05$ \\
\hline $\mathbf{3}$ & 14.52 & $<0.001$ & & $\mathbf{1 3}$ & 1.34 & NS \\
\hline $\mathbf{4}$ & 1.88 & $\mathrm{NS}$ & & $\mathbf{1 4}$ & 2.756 & NS \\
\hline $\mathbf{5}$ & 0.86 & $\mathrm{NS}$ & & $\mathbf{1 5}$ & 2.243 & $\mathrm{NS}$ \\
\hline $\mathbf{6}$ & 0.04 & $\mathrm{NS}$ & & $\mathbf{1 6}$ & 41.32 & $<0.001$ \\
\hline $\mathbf{7}$ & 0.16 & $\mathrm{NS}$ & & $\mathbf{1 7}$ & 21.57 & $<0.001$ \\
\hline $\mathbf{8}$ & 9.47 & $<0.001$ & $\mathbf{1 8}$ & 0.98 & NS \\
\hline $\mathbf{9}$ & 0.87 & $\mathrm{NS}$ & $\mathbf{1 9}$ & 0.004 & NS \\
\hline $\mathbf{1 0}$ & 7.54 & $<0.001$ & & & \\
\hline
\end{tabular}

At D.F. $3 \& 37$

\section{DisCuSSION AND CONCLUSION}

The Conclusions drawn on the basis of this clinical study conducted on 40 participants of Raktarsha are as follows:

In group A overall result was $53.88 \%$ and maximum (72.72\%) relief was observed in aadhman. In raktasrava, gudakandu, udarashula, katishula, vibandha and agnimandya result was 58.33\%, $57.14 \%, 66.66 \%, 60 \%, 52.38 \%$ and $60 \%$ respectively.

In group B overall result was $61.33 \%$ and maximum (84\%) relief was observed in raktasrava. In vibandha, gudashula, pandu and agnimandya result was $59.16 \%, 57.14 \%, 50 \%, 50 \%$, and $68.15 \%$ respectively.

In group C overall result was $55.70 \%$. In vibandha, gudankura, raktasrava, parikrtika, pandu and agnimandya result was $52.99 \%, 57.50 \%, 52.77 \%, 45 \%, 57.14 \%$ and $48.27 \%$ respectively.

$>$ In group C overall result was $65.26 \%$. In vibandha, gudankura, gudashula, arochaka and agnimandya result was $64.28 \%, 63.15 \%, 69.23 \%, 55 \%$ and $47.61 \%$ respectively.

After using ANOVA test it is found that study shows significant result in reduction in size of Piles, aadhman, raktasrava, parikartika, arochaka and agnimandya. So, this study concludes that pathadi yoga and Piccha basti together give batter result.

In group A no one was having Pravahika. In group B no one was having karshya, sangrahani and pindikodveshtana. In group $\mathrm{C}$ and $\mathrm{D}$ no one was having karshya.

\section{REFERENCES}

[1] Astanga hridayam Nidana Sthana 7/1 Page No. 476, by- Dr. Brahamanand Tripathi, Pub. Choukhambha Sanskrit Sansathan, Delhi.

[2] Charaka chikitsa sthana 14/5, Page No.417, Pndt. Kashinath Shashtri and Dr. Gorakhnath Chaturvedi. Pub. Choukhanbha Vishwabharati Varanasi.

[3] Shushruta Samhita Nidana Sthana adhyaya2/3 Page 306, By- Kaviraj Dr. Ambikadatta Shashtri, Pub. Choukhambha Sanskrit Sansathana Varanasi.

[4] Davidson's Principles and Practice of Medicine Chap.22, Page No. 915, $21^{\text {st }}$ edition, Edited by Nick R. Colledge, Brain R. Walker and Stuart H. Ralston, Pub.- Churchill LivingstoneELSEVIER

[5] Harrison's Principles of Internal medicine, By- Lonago, Fauci et al, Vol.2 $18^{\text {th }}$ edition, Chapter 297, Page No. 2507.

[6] Chakradatta, adhyaya 5/1, Page no.34, by-Vaidya Ravidutta Shashtri, Pub. Choukhambha Sanskrit Prakashana, Varanasi.

[7] Charaka Samhita chikitsa sthana14/195, Page No.442, Pndt. Kashinath Shashtri and Dr. Gorakhnath Chaturvedi. Pub. Choukhanbha Vishwabharati Varanasi.

[8] Charaka Samhita Chikitsa sthana 14/225- 229, Page No. Pndt. Kashinath Shashtri and Dr. Gorakhnath Chaturvedi. Pub. Choukhanbha Vishwabharati Varanasi. 